\title{
Del higienismo a la acupuntura urbana. Metáforas médicas y urbanismo excluyente en Bogotá
}

From Hygienism to Urban Acupuncture. Medical Metaphors and Exclusionary Urbanism in Bogotá

Do Higienismo à Acupuntura Urbana. Metáforas médicas e urbanismo excludente em Bogotá

Juan Pablo Galvis*

Recibido: 30 de septiembre de 2018

Aprobado: 2 de junio de 2019

Doi: https://www.doi.org/10.12804/revistas.urosario.edu.co/territorios/a.7297

Para citar este artículo:

Galvis, J. P. (2020). Del higienismo a la acupuntura urbana. Metáforas médicas y urbanismo excluyente en Bogotá. Territorios, (42), 1-29. Doi: https://www.doi.org/10.12804/revistas.urosario.edu.co/territorios/a.7297

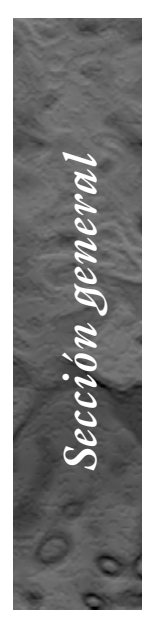

* Profesor asistente, State University of New YorkCollege at Old Westbury. Correoelectrónico:galvisj@ oldwestbury.edu. ORCID: bttp://orcid.org/00000001-7919-5063. 
Palabras clave

Planeación urbana, metáfora médica, exclusión, Bogotá.

Keywords

Urban planning, medical metaphor, exclusion, Bogotá.

Palavras-chave

Planeamento urbano, metáfora médica, exclusão, Bogotá.

\section{territarias 42}

RESUMEN

Gran parte de la literatura sobre las políticas urbanas en la Bogotá del cambio de siglo - tanto crítica como celebratoria - se concentra en caracterizar los cambios en la forma de pensar la ciudad que se dieron en esa época. Este artículo busca, en cambio, subrayar las continuidades en la forma de planear la ciudad que han reproducido un urbanismo excluyente. El artículo se centra en las metáforas médicas para entender la intervención urbana. Mostrando las continuidades del urbanismo excluyente en el urbanismo higienista de comienzos del siglo Xx, el modernismo de mediados del siglo y la revitalización urbana contemporánea, el artículo muestra cómo la exclusión reciente en procesos de revitalización urbana, de poblaciones consideradas como fuentes de degradación o enfermedad, tienen antecedentes claros en intervenciones urbanísticas del pasado. Dichas intervenciones también usaron metáforas médicas para justificar urbanismos excluyentes. El artículo sugiere que, a pesar de los repetidos fallos para producir la ciudad planificada, los diferentes paradigmas urbanísticos han logrado perpetuar un discurso que aísla áreas y poblaciones específicas como focos infecciosos y justifica su exclusión activa en el nombre de proteger la salud y bienestar común de las áreas y poblaciones "sanas".

\section{ABSTRACT}

Most of the literature about urban policy in Bogota at the turn of the century has focused on the city's transformation. Both critical and celebratory approaches have highlighted the departures from previous ways of planning and conceiving the city around that time. This article focuses instead on the continuities in Bogotá's planning as they have continued to produce exclusionary urban policy. The article focuses on medical metaphors for urban intervention, highlighting the continuity of exclusionary urbanism from early twentieth-century hygiene planning, mid-century modernism, and through contemporary urban revitalization. The article documents how the exclusion of people deemed as sources of urban malaise or deterioration in recent urban revitalization efforts harkens back to past urban interventions, which also used medical metaphors to justify such exclusions. The article suggests that despite repeated failures in producing the planned city, various planning paradigms have perpetuated a discourse that sets apart certain places and people as sources of infection and justifies their active exclusion in the name of protecting the health and wellbeing of the "healthy" city.

\section{RESUMO}

Grande parte da literatura sobre as políticas urbanas em Bogotá na mudança de século -tanto crítica quanto comemorativa- se concentra em caracterizar as mudanças na forma de pensar a cidade que se deram nessa época. Este artigo busca, por outro lado, ressaltar as continuidades na forma de planear a cidade que têm reproduzido um urbanismo excludente. $\mathrm{O}$ artigo se centra nas metáforas médicas para entender a intervenção urbana. Mostrando as continuidades do urbanismo excludente no urbanismo higienista de inícios do século $\mathrm{xx}$, o modernismo de meados do século e a revitalização urbana contemporânea, o artigo mostra como a exclusão recente em processos de revitalização urbana de populações consideradas como fontes de degradação ou doença têm antecedentes claros em intervenções urbanísticas do passado. Ditas intervenções também usaram metáforas médicas para justificar urbanismos excludentes. $\mathrm{O}$ artigo sugere que, apesar dos repetidos erros para produzir a cidade planificada, os diferentes paradigmas urbanísticos têm conseguido perpetuar um discurso que isola áreas e populações específicas como focos infeciosos e justifica sua exclusão ativa em nome de proteger a saúde e bem-estar comum das áreas e populações "saudáveis". 


\section{Introducción}

Bogotá se ha convertido para algunos en referente de cambios radicales en el gobierno del espacio público. Tanto las autoridades locales como algunos comentaristas internacionales han presentado intervenciones físicas - como la construcción de bibliotecas en zonas deprimidas, la rehabilitación de parques o corredores de transporte - como catalizadores de cambios mayores en las áreas circundantes y la ciudad en general. Igualmente, intervenciones temporales de bajo costo y de carácter artístico - como el uso de mimos para dirigir el tráfico en intersecciones concurridas y demás estrategias de lo que se conoce en Bogotá como 'cultura ciudadana' - han sido presentadas como catalizadores de cambios generales en el funcionamiento de la ciudad (Beckett \& Godoy, 2010). Según varias versiones, temas desde mejoramientos en seguridad como la reducción en la tasa de homicidios hasta una recuperación del espíritu cívico y el orgullo local son atribuibles a este tipo de intervenciones (Martin, Ceballos \& Ariza, 2004; Cervero, 2005; López, 2003).

Estudios de la forma en que la experiencia bogotana ha circulado alrededor del mundo han mostrado cómo la reputación de Bogotá ha dependido de su promoción por las autoridades locales, en confluencia con organismos privados y multilaterales dedicados a la replicación de políticas urbanas (Montero 2017a, 2017b; Duque, 2011). Estos métodos han sido teorizados como 'Acupuntura Urbana' (Solà-Morales, 2008; Lerner, 2014; Casanova \& Hernández, 2015) 'Urbanismo Táctico' (Burdett, Cruz, Harvey, Sassen \& Tehrani, 2014; Lydon, García \& Duany, 2015) o 'Placemaking' (Gehl, 2010; Brown, Dixon \& Gillham, 2009; Thomas, 2016; Walljasper, 2007), entre otros, y se han popularizado durante la última década como soluciones de bajo costo contra los más variados y persistentes problemas urbanos. La promoción de Bogotá como ejemplo de estas tácticas ha subrayado dichas políticas. En este contexto, la ciudad ha sido objeto de análisis académico para entender el verdadero alcance de sus políticas. Varios estudios celebran los cambios en la cantidad y calidad del espacio público en la ciudad, así como los elementos políticos y urbanísticos de su transformación (Beccassino, 2000; Borbón, 2003; Martin, 2007; Berney, 2011, 2017). Sin embargo, análisis críticos han mostrado cómo la reputación de la ciudad como modelo ha tendido a ignorar los mecanismos de exclusión social implementados en nombre de espacios públicos supuestamente incluyentes (Ritterbusch, 2016; Galvis, 2014, 2017a; Muñoz, 2018). De hecho, dichos análisis subrayan las contradicciones del ejemplo bogotano, mostrando cómo la revitalización de sus espacios públicos incluyó, al igual que en otros muchos lugares, el desplazamiento de poblaciones vulnerables, en particular habitantes de territarias 42 
calle y vendedores ambulantes (Galvis, 2017b; Donovan, 2008, 2010).

Más que definir si Bogotá puede o no ser considerada un modelo de alguna política urbana, este artículo usa el caso de la ciudad para analizar la persistencia de las exclusiones documentadas en la literatura. El artículo historiza las intervenciones recientes, en el marco de al menos un siglo de intervención urbana en la ciudad. Para esto, se centra en el uso de metáforas médicas para entender la intervención urbana. En este sentido, el artículo propone que las exclusiones producidas en Bogotá pueden ser entendidas como elementos inherentes a la intervención médica y parte de una larga tradición de intervención urbana excluyente que ha usado la metáfora médica como elemento de su justificación técnica y política.

El artículo analiza cómo las metáforas médicas en la planeación urbana han servido para replicar la marginalización y negación del derecho a la ciudad de determinadas poblaciones. Tras una contextualización teórica de las metáforas orgánicas para entender la ciudad y sus correspondientes metáforas de intervención médica, el artículo revisa tres paradigmas de la planificación en Bogotá: El higienismo de comienzos del siglo $\mathrm{xx}$, el modernismo de mediados del siglo y el urbanismo contemporáneo del fin de siglo. Para ello, indaga cómo la exclusión de poblaciones consideradas agentes de enfermedad o 'degeneración' ha sido una constante en la planeación urbana de
Bogotá en estos tres periodos. El uso de metáforas médicas, de hecho, ha servido para justificar la exclusión de dichas poblaciones en nombre del bienestar común. En este sentido, el artículo busca dar un contenido histórico a las intervenciones en el espacio público en Bogotá. En mucha de la literatura sobre el proceso de transformación de la ciudad alrededor del cambio de siglo - tanto crítica como celebratoria- se describe una transformación radical en la forma de concebir la ciudad y una etapa de ruptura con el pasado (Berney, 2017). El artículo busca en cambio dar cuenta de las continuidades en varios aspectos de la planificación urbana. Al analizar los ecos de metáforas médicas en políticas de planificación del pasado, el artículo muestra algunas de las constantes que han producido - $-\mathrm{y}$ continúan produciendo- la exclusión de diversas poblaciones del espacio urbano.

\section{Metáforas médicas: el urbanismo como intervención curativa}

El uso de metáforas médicas para entender la planificación urbana no es nuevo. De hecho, estas pueden considerarse como parte de una amplia corriente que entiende la ciudad como organismo vivo, cuyos problemas son el resultado de patologías y desarreglos orgánicos. Así, la planeación se ve como un tratamiento medicinal para curarlos. De este modo, los tres paradigmas urbanísticos considerados en este artículo son analizados según su uso 
de metáforas orgánicas para entender la ciudad. Las diferentes versiones de estás metáforas implican la construcción paralela de metáforas médicas para entender la intervención urbana como tratamiento. En el caso del higienismo, por ejemplo, la intervención urbana, estructurada como intervención curativa o preventiva de enfermedades, hace parte de un entendimiento orgánico de la ciudad como cuerpo social y de ciertas clases sociales como elementos patológicos. En el caso de la acupuntura urbana, la ciudad se entiende como un cuerpo orgánico desbalanceado que necesita de intervenciones puntuales revitalizadoras que catalicen una recuperación urbana generalizada. $\mathrm{Al}$ mismo tiempo, esta revitalización se centra en lugares ocupados por habitantes o usos considerados inapropiados.

Las analogías entre realidades sociales y biológicas han servido repetidamente para racionalizar la exclusión o subordinación de diversas capas sociales (Canguilhem, 2008). Por ejemplo, Aristóteles proponía a la ciudad como "una criatura natural con vida propia" (Ambler, 1985, p. 169), con el objetivo de naturalizar las jerarquías sociales como parte del 'cuerpo' orgánico de la ciudad (Kraut, 2002, p. 366). Ejemplos de este uso incluyen la dominación de clases o razas particulares o, como fue el caso del Darwinismo Social y el Racismo Científico, la colonización, la eugenesia y el genocidio (Hawkins, 1997; Sánchez, 2008).
El gobierno urbano no ha sido ajeno a esta tendencia. La justificación de urbanismos excluyentes desde el urbanismo colonial europeo del siglo XIX hasta la segregación racial del siglo XX estuvo al menos en parte fundamentada en la necesidad de preservar la buena salud de la ciudad (Goldberg, 1992). De hecho, según Foucault (2008, 2009), símiles entre la ciudad y un organismo vivo fueron recurrentes en el desarrollo de la biopolítica moderna. En la ciudad, sugiere, se concentran tales "problemas $[. .$.$] de$ una coexistencia densa" como "la salud, la subsistencia, los medios para impedir la escasez, la presencia de mendigos [y] vagabundos" (2008, p. 318). Así, el objetivo del gobierno es buscar la buena salud y seguridad del territorio y la población de la ciudad. De hecho, muchas de las técnicas básicas para el desarrollo de la biopolítica fueron de naturaleza médicobiológica. Políticas sanitarias destinadas a mejorar la salud pública están entonces apoyadas en ideas sobre la ciudad como cuerpo orgánico necesitado de tratamientos específicos.

La planificación urbana de la segunda mitad del siglo XIX ilustra este punto bastante bien. En respuesta al rápido despliegue de enfermedades contagiosas como el cólera, la tuberculosis y, en ciudades tropicales, la fiebre amarilla y la malaria, varias técnicas de planeación urbana elevaron la sanidad como concepto rector de la intervención urbana. Sin embargo, el llamado 'higienismo', con ejemplos tanto 
en la ciudad industrializada de Europa y Estados Unidos (Shah, 2001; Johnson, 2007) como en las ciudades coloniales asiáticas y africanas (Anderson, 2006; Bigon, 2016; Gandy, 2014) y en las ciudades latinoamericanas de comienzos del siglo Xx (Kingman. 2006; Armus, 2007; Ibarra, 2016), fue notorio no solo por su interés en prevenir la diseminación de enfermedades contagiosas. De hecho, las intervenciones urbanas higienistas usaban el conocimiento técnico y la justificación política de la salud pública para reordenar el espacio urbano en formas que o bien marginalizaron a clases populares racializadas y sujetos coloniales o bien buscaban transformar el comportamiento e incluso las características biológicas de estos (Holston, 2008). En la mayoría de las ciudades latinoamericanas, dichas intervenciones fueron adaptadas por las élites locales a ciudades relativamente pequeñas y no industriales. Así, las curas de la ciudad higienista fueron aplicadas localmente tanto para combatir verdaderas epidemias como para implementar ideales acerca de las transformaciones morales y físicas necesarias para producir ciudadanos sanos y civilizadas (Castro-Gómez, 2009).

Hacia mediados del siglo $\mathrm{xx}$, la planificación higienista dio paso a nuevos paradigmas de planificación urbana. El movimiento modernista del CIAM fue particularmente influyente a este respecto en las ciudades latinoamericanas. En vez de buscar reformar sujetos sanos y civilizados, el urbanismo modernista buscaba regular el funcionamiento urbano mediante técnicas como la zonificación y la regulación de flujos, entre otros (Mumford, 2002). A pesar de los cambios que el movimiento modernista introdujo en la teoría y práctica de la planificación urbana, esta continuó usando analogías biológicas para entender la ciudad. En pocos es esto más evidente que en Josep Lluís Sert, discípulo de Le Corbusier y figura prominente del movimiento modernista en la segunda mitad del siglo. En su libro Can Our Cities Survive: An abc of urban problems, Sert (1942) propuso que "para conocer nuestras ciudades de manera completa [... podríamos considerarlas como organismos vivos; cosas que nacen, se desarrollan, se desintegran y mueren" (p. 3). Para Sert, el objetivo de la planificación moderna es propender por la sobrevivencia de las ciudades. Esto requería de la aplicación de la racionalidad y el conocimiento científico para tratar los problemas urbanos. Según Sert (1942), "la planeación urbana se ha vuelto obsoleta. Debe ser reemplazada por la biología urbana o el estudio de la vida de las ciudades" (p. 3 ).

Sin embargo, el discurso modernista no se apartó del todo de las prescripciones higienistas. Sus intervenciones a gran escala también tendieron a curar la ciudad mediante el desplazamiento de sus habitantes más marginales. En la Carta de Atenas, por ejemplo, Le Corbusier (1973) anunciaba cómo en nombre de "las leyes de la higiene [y] de la salud pública deberían ser condenados barrios 
enteros $[\ldots]$ destruir sin piedad cuanto constituye un peligro" (p. 62), Ejemplos de esto - así como de la resistencia en su contra- abundan tanto en Norteamérica (Estrada, 2005; Mohl, 2004) como en América Latina (Sagaris, 2014; Holston, 2008).

Las metáforas médicas sobre la ciudad no se han limitado al urbanismo propiamente dicho. Otros tipos de conocimientos sobre el funcionamiento social urbano también han hecho uso repetido de analogías sobre la ciudad como organismo vivo y de sus problemas como patologías (Nye, 1985). Entre las más notables está la sociología urbana, que organizó su análisis de la ciudad alrededor de lo que llamaron la "vida metropolitana" (Simmel, 2004). Según esta visión, los problemas urbanos eran patologías atribuibles tanto al espacio como a los habitantes urbanos y requerían intervenciones sociales a modo de tratamiento científico. Muchas intervenciones modernistas como la construcción de grandes bloques habitacionales para familias de bajos ingresos $-\mathrm{y}$ su posterior demolición - han sido llevados a cabo acudiendo tanto a discursos urbanísticos sobre la ciudad como organismo vivo, como a discursos sociológicos sobre patologías de las poblaciones urbanas (Crump, 2002).

Un claro ejemplo contemporáneo de esta aproximación es la llamada "teoría de las ventanas rotas" (Kelling, 1996). Esta influyente teoría sugiere que diversas patologías urbanas de menor importancia - como la presencia de ventanas rotas, grafiti y otras transgresiones menoresproducen un efecto contagio que resulta en patologías peores (Ludwig \& Kling, 2007). En resumen, esta teoría sugiere que mediante el tratamiento directo de dichas patologías se puede combatir el problema mayor de la criminalidad urbana.

Esta teoría y sus métodos de intervención urbana no se hicieron esperar en varias ciudades latinoamericanas, donde la asociación entre poblaciones específicas como los vendedores ambulantes y otros supuestos agentes de criminalidad y desorden no era nueva (Swanson, 2007, 2013; Galvis, 2014). En Bogotá se hizo referencia explícita a este tipo de intervención urbana como una forma de recuperar la ciudad enferma del desorden producido, en teoría, por dichas infracciones no criminales (Alcaldía de Bogotá, 2000).

Más recientemente, otro cambio de paradigma urbanístico ha desplazado el discurso modernista sin abandonar la metáfora médica. Desde los primeros críticos del urbanismo modernista como Jane Jacobs (1961) y Kisho Kurokawa (2000), hasta urbanistas contemporáneos como Jan Gehl (2008), han basado sus propuestas en metáforas sobre la ciudad como un organismo vivo y el urbanismo como terapia. Estos críticos del modernismo insisten en que su racionalismo a gran escala esterilizó la ciudad, privándola de su vitalidad. Así, vibrantes ciudades se transformaron en apacibles sectores residenciales, asépticas zonas de oficinas y centros comerciales cerrados. Su 
prescripción consiste en devolverle la vida a la ciudad — es decir, su "revitalización"— mediante el rescate de los espacios públicos y la creación de múltiples centralidades de uso mixto. Comúnmente centradas en la revitalización de centros urbanos, estas propuestas sugieren que la vitalidad urbana puede ser estimulada mediante la activación de espacios públicos puntuales con actividades artísticas, ferias y demás eventos que inviten a la población a usar los centros y otras áreas desvalorizadas.

Uno de los mejores ejemplos del urbanismo contemporáneo como antídoto contra los excesos del modernismo es la acupuntura urbana (Lerner, 2014; Casagrande, 2015; Casanova \& Hernández, 2015; Salazar, Mojica \& Urrea, 2015; Solà-Morales, 2008). Según el urbanista brasileño Jaime Lerner (2014) la metáfora sugiere la aplicación de:

[...] 'magias' de la medicina a las ciudades, pues muchas están enfermas [...]. Del mismo modo en que la medicina necesita la interacción entre el médico y el paciente, en urbanismo también es necesario hacer que la ciudad reaccione. Tocar un área [para] curar, mejorar, crear reacciones $[\ldots][\mathrm{e}] \mathrm{s}$ necesario intervenir para revitalizar (p. 1).

Sin embargo, la enfermedad urbana está en este caso asociada con el énfasis del modernismo en la zonificación y la circulación vehicular. Lerner (2014) sugiere, por ejemplo, que el "colesterol urbano, la acumulación en nuestras venas y arterias del uso excesivo del automóvil" (p. 95) es producto de la construcción de ciudades en función de la circulación entre diversas zonas.

A modo de cura al autoritarismo tecnocrático a gran escala del modernismo, la acupuntura urbana propone un urbanismo a escala humana. Casanova y Hernández (2011), por ejemplo, proponen la "regeneración de la vida pública" mediante la “acupuntura del espacio público," una serie de "pequeñas intervenciones que regeneren el espacio público urbano y la vida de la ciudad" mediante la incorporación participativa de las comunidades circundantes (p. 11). Esta regeneración de la vida urbana barrial se concibe como contagiosa, generando efectos sobre la ciudad en general. En vez de soluciones de circulación, las autopistas modernistas son concebidas como grandes "heridas" o "fracturas" que deben ser "suturadas" para volver a establecer conexiones de escala barrial en el "tejido urbano".

Interpretaciones críticas sobre este tipo de intervención no se han hecho esperar (Peck, 2005; Crouch, 1998; Shatkin, 2011). Dichas críticas apuntan hacia estas terapias como políticas propiciadoras de la gentrificación. Una copiosa literatura muestra cómo alrededor del mundo los esfuerzos de revitalización urbana han creado las condiciones para el desplazamiento de poblaciones vulnerables de lugares atractivos para el redesarrollo de proyectos inmobiliarios de lujo y su consiguiente ocupación por capas de 
altos ingresos (Atkinson \& Gary, 2005; Janoschka, Sequera \& Salinas, 2014). Así, la revitalización o regeneración de zonas deterioradas ha estado acompañada del desplazamiento de diversas poblaciones (Sevilla, Castrillo, Matesanz \& Sánchez, 2014). De hecho, la mera presencia de estas poblaciones es considerada como una de las principales señales de deterioro, de manera que su revitalización pasa necesariamente por el desplazamiento de personas asociadas con patologías urbanas como la criminalidad o el desorden.

Como se ve, las metáforas bio-médicas para entender la ciudad y justificar políticamente su intervención han circulado ampliamente en la teoría y la práctica de la planeación urbana en diversos contextos históricos y geográficos, sobreviviendo a varios cambios de paradigma urbanístico. Desde el urbanismo higienista hasta la acupuntura urbana, la metáfora médica ha sido parte importante de dichos paradigmas. Al mismo tiempo, como lo indican varias literaturas críticas, la metáfora médica ha servido como herramienta de justificación política de intervenciones tecnocráticas y excluyentes de diversas poblaciones. En el caso del higienismo, el objetivo médico consistía en la segregación o el mejoramiento de razas u otros grupos poblacionales considerados como focos de enfermedad o degeneración. En el caso del modernismo, la intervención a gran escala para zonificar la ciudad y mejorar la circulación urbana, buscó reformar los barrios populares, vistos bien como cánceres que afectaban a la ciudad en su conjunto o bien como la línea de menor resistencia para desembotellar la circulación. Finalmente, la acupuntura urbana y otros paradigmas similares usan metáforas médicas para conceptualizar el mejoramiento del espacio público y la programación de actividades para atraer de nuevo la vida a la ciudad. Sin embargo, dicha revitalización a menudo deviene en la gentrificación de espacios urbanos considerados patológicos y tiende a privilegiar el disfrute del espacio público de propietarios y transeúntes en detrimento de otros posibles usos por parte de poblaciones vulnerables. En la siguiente sección, el artículo explora estas continuidades en Bogotá, para documentar la persistencia de prácticas y discursos excluyentes.

\section{Metáforas médicas y planeación en Bogotá}

En esta sección, el artículo explora la continuidad en Bogotá de las metáforas médicas explicadas anteriormente. En particular, muestra cómo desde el higienismo de inicios del siglo Xx hasta la acupuntura urbana contemporánea, las diferencias en el paradigma planificador no han modificado el uso de la metáfora médica para identificar varias poblaciones como focos de enfermedad urbana y justificar su exclusión en nombre de la buena salud de la ciudad (Robledo \& Rodríguez, 2008).

$\mathrm{Al}$ igual que en muchas otras ciudades latinoamericanas, el urbanismo higienista 
apareció en Bogotá en la segunda mitad del siglo XIX. Si bien la ciudad no enfrentaba los problemas sanitarios de las ciudades industriales, sí precisaba de regulaciones sobre la distribución de agua potable y la localización de lugares sanitariamente sensibles. Así, los primeros acuerdos municipales que incorporaron asuntos de higiene pública al planeamiento de la ciudad tuvieron que ver con la provisión de aguas y la localización del matadero y el cementerio (Concejo de Bogotá, 1865, $1880,1881)$. Estas iniciativas continuaron con la creación de la Junta de Higiene y la Dirección de Higiene y Salubridad, las primeras instituciones dedicadas al control urbano dirigido a lo que los acuerdos municipales de la época llaman "el ornato, salubridad y seguridad" de la ciudad. Si bien la creación de estas instituciones respondía a necesidades inmediatas de, entre otras cosas, controlar enfermedades contagiosas, también reflejaba un interés entre las élites de la época por producir una ciudad civilizada y de progreso. De esta forma, referencias a la sanidad y el ornato de la ciudad comprendían ideas mucho mayores sobre las condiciones de la deseada ciudad civilizada (Castro-Gómez, 2009). Así mismo, el urbanismo higienista hacia parte de dispositivos de exclusión y control social dirigidos a marginalizar, controlar o reformar las racializadas clases populares (Robledo \& Rodríguez, 2008; Noguera, 2003; Castro-Gómez, 2009).

Varios estudios sobre el planeamiento

territarias 42 cómo las políticas higienistas no fueron simples lineamientos técnicos políticamente neutrales, dirigidos estrictamente a temas sanitarios (Noguera, 1998, 2003, Suárez, 2006). De hecho, el higienismo marcó una época en la construcción de políticas urbanas desde temas urbanísticos como la vivienda y el desarrollo vial hasta temas sociales relacionados con la pobreza y la criminalidad (Ceballos, 2008; Mejía, 2000; Zambrano, 2009). Así, desde las primeras visiones modernas de una ciudad higiénica, se empezaron a desarrollar prescripciones sobre la disposición de viviendas populares, construcción de acueductos, avenidas, plazas y parques (Castro, 2013; Colón, 2005). El diario El Tiempo reportaba en 1911 , por ejemplo, cómo "los médicos de la Oficina de Salubridad y Beneficencia" sugerían una serie de medidas sanitarias como la construcción del "acueducto, matadero, plazas de mercado, [...] escuelas, habitaciones para obreros, hospitales y prisiones" los cuales significarían un "gran paso hacia la civilización y hacia la libertad" (El Tiempo, 1911). Como se ve, la higiene no estaba dirigida exclusivamente a patógenos contagiosos o aguas residuales. Incluía además temas de educación, vivienda popular, vías y hasta prisiones.

Estas ideas evolucionaron en los círculos profesionales de la época, superponiendo discursos sobre la higiene urbana con conocimientos médicos sobre las enfermedades contagiosas y la llamada degeneración de la raza (Noguera, 2003). 
De este modo, la intervención médica justificó actuaciones urbanas que apuntaban de manera bastante explícita hacia poblaciones y comportamientos que debían ser sujetos de intervención para conseguir una ciudad higiénica. En entrevista con El Tiempo en 1929, por ejemplo, el Dr. Alejandro Villa de la Dirección de Higiene dejaba clara la diversidad de problemas asociados con la higiene que precisaban de intervención médico-científica: "las aguas, las calles, la chicha, la pobreza de nuestras clases inferiores y su falta de educación" (El Tiempo, 1929).

Entre los ejemplos de intervenciones urbanas higienistas se cuentan los esfuerzos por "sanear" el Paseo Bolívar y la construcción de la Avenida Jiménez de Quesada. La zona del Paseo Bolívar, en el borde oriental de la ciudad, era notoria hacia la década de 1910 como uno de los centros de concentración de pobreza en Bogotá. Las "clases inferiores" eran descritas entonces en términos de la falta de higiene de sus viviendas. Como lo muestra Colón (2005), en especial tras la epidemia de gripa de 1918, médicos y urbanistas se centraron en esta zona "infecta y desaseada” (Colón 2005, p. 108), desarrollando diversos planes para su saneamiento. Dichos planes usaban el discurso higienista para justificar el desplazamiento de la población de estos barrios que, al ubicarse aguas arriba del centro tradicional, eran vistos como una amenaza sanitaria para la ciudad en su conjunto.
Sin embargo, estos planes higienistas no solo comprendían la extirpación de los elementos anti-higiénicos de la ciudad construida. Eran también planes para "reformar material y moralmente el estado de las clases trabajadoras" que las habitaban (Zambrano, 2009, III: p.173). Junto con la demolición de las viviendas populares, se contemplaba la construcción de nuevas "habitaciones obreras," aguas abajo, según reglas higiénicas y alejadas del centro urbano. La construcción de estos barrios debía evitar la concentración de clases populares en grandes barrios obreros a fin de prevenir la construcción de "focos de desorden [y la] formación de una casta hostil" (Colón, 2005, p. 110). Para los higienistas, la concentración de clases populares en grandes barriadas obreras no solo significaba una amenaza sanitaria para la ciudad en su conjunto. Sin intervención higiénica, estas clases constituían una amenaza social y política y representaban focos de desorden. Como se verá más adelante, este discurso que focaliza la intervención sobre diversos sujetos urbanos inherentemente desordenados o peligrosos ha sobrevivido en la planeación de Bogotá (Suárez, 2006).

Una lógica similar sirvió de base para la canalización del río San Francisco, zona que en 1917 era descrita como "un peligro inminente para la salud [por ser el polvo y el lodo] vehículos eficacísimos para la propagación de las peores epidemias" (Concejo de Bogotá, 1917). El proyecto contemplaba la construcción de la Avenida territarias 42 
Jiménez que, según la prensa de la época, podría "rivalizar con las mejores calles europeas" y requería "transformar las horribles y malsanas ruinas que [forman] el cauce del río San Francisco" (Cuéllar \& Mejía 2007, p. 510), así como regular las nuevas edificaciones que serían construidas a lado y lado de la vía, prohibiendo "la construcción de chicherías, cuarterías, pesebreras y carbonerías y el 'desalojo' inmediato de las existentes" (Cuéllar \& Mejía, 2007, p. 511).

Regular la salud de la ciudad era para el higienismo tanto sobre el cuerpo físico como el cuerpo social urbano. Ambos eran entendidos según discursos médicobiológicos. Según el Dr. Villa, la intervención urbana higienista, "como ocurre en todos los países civilizados, [representa] el símbolo vivo del interés social en el mejoramiento de la raza". Dicho mejoramiento racial estaba ligado directamente a un esfuerzo civilizatorio dirigido específicamente a "[corregir] aquellos defectos inherentes a nuestra idiosincrasia [con una] gran obra de enseñanza y de moralización luchando contra los prejuicios, los hábitos funestos, las enfermedades y la ignorancia" (El Tiempo, 1929). Hacia esta época, el urbanismo higienista en Bogotá se concentró específicamente en el combate específico de varios de los "hábitos funestos" de las clases populares, el más notorio de los cuales fue el consumo de chicha (Calvo \& Saade, 2002). Los médicos relacionaron el consumo de territarios 42 chicha con la "completa decadencia" y "descendencia degenerada" de "las razas Aborígenes de América” (Torres, 2001, p. 130). De igual forma, esta degeneración se asociaba con otros problemas urbanos como la criminalidad.

Las autoridades municipales buscaron contener dicha degeneración mediante medidas urbanísticas para curar el cuerpo social urbano. El primer acuerdo municipal de 1916 que reguló la fabricación y venta de chicha en Bogotá, dejaba abundantemente clara la conexión entre el discurso higienista y las mencionadas ideas sobre raza y civilización prevalentes entre las autoridades de la época. El Concejo Municipal justificó la regulación de la chicha, considerando:

[...] los estragos que causa el alcoholismo en [...] la moral y la salubridad públicas y la degeneración visible de la raza, que "la ciencia demuestra que los hijos de los alcoholizados son seres degenerados, incapaces para el trabajo y agentes en embrión de crímenes o delitos" y "que es deber imperioso del Concejo velar por la salud y moralidad del pueblo, [...] para que no degenere el vigor de la raza" (Concejo de Bogotá, 1916).

La regulación de la chicha fue un claro uso de la justificación médica para atacar las costumbres populares que se identificaban con la "degeneración de la raza" y la falta de civilización y se asociaban con otras patologías urbanas como la criminalidad y el posible contagio que de las barriadas 
populares pudiera surgir hacia áreas centrales o suburbios pudientes. De hecho, las reglas sobre chicha expedidas por el Concejo Municipal en 1916 y luego entre 1922 y 1928 (Concejo de Bogotá 1922a, 1922b, 1928), zonificaron el expendio y fabricación de chicha fuera del centro y el naciente suburbio de Chapinero. El objetivo era limitar los supuestos efectos “degeneradores" a los barrios periféricos, aislando las zonas centrales y suburbios residenciales de clase media y alta.

De este modo, intervenciones físicas como el saneamiento del Paseo Bolívar y la canalización del río San Francisco se articularon con intervenciones de control urbano como la regulación de la chicha, formando discursos sobre la intervención médica de la ciudad. En ambos casos, la ciudad estaba enferma debido a la falta de civilización y la degeneración racial de las clases populares, cuya reforma y/o exclusión de la ciudad central era necesaria en beneficio del bienestar general de la ciudad.

Si bien el higienismo dio paso al urbanismo modernista hacia mediados de siglo, muchos de estos discursos excluyentes fueron transferidos a este nuevo paradigma. No es coincidencia, por ejemplo, que la Ley 88 de 1947, la misma que ordena la expedición de planes reguladores - la herramienta esencial del urbanismo modernista - prohibiera también a nivel nacional el expendio de chicha y guarapo cerca de áreas centrales (Colombia, 1947). Igualmente, a nivel local, la adopción formal de dicho plan regulador en 1951 se justificó como la manera más adecuada de "mejorar la salud pública, la seguridad y el bienestar de los habitantes" (Alcaldía de Bogotá, 1951).

En el caso de Bogotá, la máxima expresión de la planificación modernista se dio con la formulación del plan director y el plan regulador de Sert y Le Corbusier hacia finales de los años 40 (Tarchópulos, 2006; O'Byrne, 2010). Estos planes, sin embargo, se formularon sobre la base de iniciativas anteriores como el Plano Bogotá Futuro y los planes desarrollados durante las décadas anteriores por Karl Brunner, arquitecto del ensanchamiento de la Bogotá de los años treinta y, entre otros, impulsor del plan para la renovación del Paseo Bolívar (Colón, 2005; Castro, 2013). De hecho, el cambio hacia la planificación funcional modernista en Bogotá fue en gran parte el resultado de la adaptación de algunas de las iniciativas higienistas, en especial las relativas a la construcción de vivienda obrera (Hofer, 2003; Almandoz, 2007). Brunner, junto con varios otros actores locales, enfatizó en sus planes la necesidad de usar técnicas modernas para el "saneamiento" de la ciudad, refiriéndose al trabajo del urbanista francés Marcel Poëte, cuyas teorías estaban repletas de metáforas organicistas para entender la ciudad (Arango, 2018). Ciertamente, en los años anteriores a la llegada de modernistas de renombre como Sert y Le Corbusier, urbanistas modernistas locales venían proponiendo planes 
de reforma modernista. Por ejemplo, desde la revista Proa — principal órgano de difusión de la arquitectura moderna en Colombia por esa época- proponían que "Bogotá puede ser una ciudad moderna" con una "red de grandes arterias necesarias al sistema circulatorio" para producir “economía, alegría [...] luz [e] higiene" (Amorocho, García, Angulo \& Martínez, 1946 , p. 15). Igualmente, modernistas locales como Carlos Martínez, que ejercerían mucha más influencia en la planificación modernista de Bogotá en las décadas posteriores al plan regulador, sirvieron como puente entre el modernismo de Sert y Le Corbusier y la "evolución urbana" de Marcel Poëte (Arango, 2019). Dichos urbanistas locales buscaron, al mismo tiempo, conectar estos cambios de paradigma urbanístico con tradiciones nacionales (Mondragón, 2008).

La "biología urbana" propuesta por Sert en Bogotá recogía varias de las prescripciones de la planificación higienista transferidas a los principios de la Carta de Atenas (Schnitter, 2007). En palabras del mismo Le Corbusier, estos principios mandaban la curación de los "leprosos suburbios" para "darle vida al organismo" urbano (Mumford, 2007). Según lo documenta Mumford (2007), estos principios debían "integrar las estructuras sociales con las nuevas tecnologías dentro de un 'cuerpo racional', [buscando] la 'armonía biológica' [...], con la vivienda individual como 'célula' básica del 'organismo’ metropolitano” (p. 108). Si bien el modernismo prescindía de discursos sobre la degeneración racial o moral de poblaciones particulares, las metáforas médicas sí apuntaban a transformaciones sociales y físicas en la ciudad. De esta forma, discursos sobre civilización y mejoramiento racial fueron reformulados en términos de progreso y desarrollo urbano, con el urbanismo moderno como terapia de choque.

La vivienda popular, "célula básica" de la ciudad, continuó siendo un área de intervención crucial tanto en los planes directores de modernistas extranjeros como en proyectos específicos de modernistas locales (Goossens, 2014). Durante el higienismo, los barrios populares representaban la evidencia física de la degeneración racial. Para los modernistas, la construcción de vivienda en altura para reemplazar dichas barriadas debía servir para abrir espacio a las grandes avenidas, centros administrativos y monumentales que habrían de organizar la vida urbana. De esta forma, los modernistas proponían la demolición de barrios enteros - los "leprosos suburbios" de Le Corbusierpara dar paso a nuevos rascacielos habitacionales u otras intervenciones a gran escala. Esta no fue la excepción en Bogotá, donde los arquitectos modernistas propusieron la demolición de buena parte de la ciudad construida para dar paso a habitaciones en altura.

Dado su alto costo, la mayoría de los planes a gran escala ideados por los urbanistas modernistas para reformar la ciudad enferma nunca fueron ejecutados en su 
totalidad. En Bogotá, los escasos planes de vivienda popular evitaron la construcción en altura y se siguieron desarrollando en las afueras de la ciudad y según los preceptos higienistas (Saldarriaga, 1995, 1996). De igual modo, los barrios populares, en su mayoría desarrollados informalmente, siguieron siendo vistos como un "cáncer congénito del proceso de urbanización" que requería de la intervención planificadora dado su potencial para "producir grandes perturbaciones en la ciudad" (ICT, 1964 , p. 5 ). Llamados a "erradicar" tugurios e invasiones, para proteger la buena salud de la ciudad, instituciones como la Caja de Vivienda Popular y el Instituto de Crédito Territorial desarrollaron diversos planes de reubicación de barrios de invasión hacia zonas periféricas en el sur y occidente de la ciudad. Por ejemplo, en 1966, previo a la construcción de la facultad de medicina de la Universidad Nacional, las autoridades promovieron la desocupación violenta del barrio Policarpa Salavarrieta.

Si bien muchos de los planes modernistas para la construcción de vivienda a gran escala nunca se completaron, su legado es más evidente en la construcción de sistemas de circulación con jerarquías viales (Tarchópulos, 2006). De hecho, los planificadores locales centraron la mayoría de sus esfuerzos, desde finales de los años cuarenta, en la apertura o ensanchamiento de vías como arterias vitales para la ciudad moderna, incluyendo la Carrera Décima y la Avenida de los Cerros (Acebedo \&
Moreno, 2003). Para los modernistas, la circulación vial era de vital importancia para la buena salud de la ciudad, pues permitía la racionalización de los flujos del organismo urbano. Al igual que en muchas otras partes del mundo, la construcción de dichos ejes viales implicó en Bogotá el desplazamiento o segregación de barrios marginales (Torres, 2013).

Siguiendo esta tendencia, y tras tres décadas de su "saneamiento", el Paseo Bolívar fue nuevamente objeto de planes para una intervención urbanística con la construcción de la Avenida de los Cerros, una autopista urbana que extendería su trazado hacia el norte y el sur. La avenida era el eje principal del Programa Integral para el Desarrollo Urbano de la Zona Oriental de Bogotá (PIDUZOB), formulado a comienzos de los setenta, como herramienta de intervención de la zona que concentraba buena parte de los barrios populares de la ciudad. Esta vez, los urbanistas buscaban más que mejorar la circulación urbana (Everett, 1998). Allí, además del buldócer que se convertiría en símbolo del autoritarismo modernista $-\mathrm{y}$ continuaría siendo celebrado como instrumento de desarrollo por los planificadores modernistas por décadas (Acebedo \& Moreno, 2003) - la extirpación del "cáncer congénito" sería también producto de presiones inmobiliarias.

El PIDUZOB fue objeto de enconada resistencia por parte de sectores opuestos al desalojo de barrios populares y al modelo de financiamiento (Torres, 2013; 
Chaparro, Pulido y Mendoza, 1997). Enfrentando esta resistencia, y articulando los ecos del higienismo, el director de planeación insistía en 1972 que "la expulsión de las clases populares originada por la Avenida de los Cerros es un factor positivo [y] de progreso para la ciudad" (citado en Grupo de Estudios José Raimundo Russi, 1975, p. 74). También en defensa del PIDUZOB, Fernando Wiesner, presidente de la Sociedad Colombiana de Arquitectos y uno de los principales exponentes del urbanismo modernista en la ciudad, anotaba en 1973 que el plan era un ejemplo de "urbanismo curativo [que] viene a aliviar déficts" y de "urbanismo preventivo [que prevé] la ciudad del futuro" ( $E l$ Tiempo, 1973a). Coincidencialmente, el diario El Tiempo reportaba el mismo día la continuada "campaña contra la "chicha' en los barrios," anunciando "batidas contra los 'chicheros', [pues] el creciente consumo perjudica a los trabajadores y [sus] familias" (El Tiempo, 1973b). Las curas higienistas y modernistas confluían a comienzos de los setenta en los barrios marginales, cuyas poblaciones representaban enfermedades urbanas sujeto de intervención curativa.

El paradigma del urbanismo modernista dio paso a mediados de los años noventa a tendencias centradas en el espacio público peatonal como área básica de intervención para revitalizar espacios urbanos deteriorados. Estas innovaciones fueron especialmente claras a partir de las alcaldías de Antanas Mockus y
Enrique Peñalosa entre 1995 y 2003. La metáfora médica en este caso describe la intervención para recobrar la vitalidad de zonas deterioradas. Articulando debates sobre la "vitalidad urbana," por ejemplo, Rodríguez, Jolly y Niño (2004, p. 27) caracterizaban el deterioro urbano en Bogotá como el "empeoramiento o disminución de las condiciones sanas de un sitio".

Estas "condiciones sanas" han estado estrechamente ligadas a políticas de seguridad urbana. De hecho, los paradigmas de la sociología urbana que entienden la criminalidad urbana como patología fueron instrumentales en esta época para entender la revitalización, particularmente en lugares centrales de la ciudad. Así, por ejemplo, el Plan Especial de Manejo y Protección del Centro Tradicional (PEMP), la más reciente de las políticas orientadas a áreas centrales, demanda la "revitalización de la zona agobiada por la inseguridad el deterioro y el vandalismo" (El Espectador, 2016). La revitalización ha ido de la mano con políticas de seguridad, a su vez diseñadas como políticas de salud pública dirigidas a tratar los factores de riesgo asociados con patologías urbanas como la criminalidad y el deterioro (Llorente, 2005). Por ejemplo, las autoridades distritales hablaron, desde mediados de los noventa, de "políticas saludables para la seguridad y convivencia" e implementaron campañas de "vacunación contra la violencia" (Acero, Vargas, Bulla \& Cardona, 1997). 
En cuanto a la revitalización física, una de las intervenciones más ambiciosas en este periodo comprende una serie de planes de revitalización del centro de la ciudad, incluyendo el Plan Zonal del Centro (PZC-2007) y, más recientemente, el Plan de Revitalización del Centro Tradicional (2015) y el PEMP (2017). Este último, por ejemplo, según el director del Instituto Distrital de Patrimonio Cultural - la agencia a su cargo- busca rescatar en "el centro tradicional $[\ldots]$ un corazón competitivo, seguro y atractivo [pues] una ciudad sin corazón, como organismo vivo, no vive" (Alcaldía de Bogotá, 2017).

A diferencia de las grandes intervenciones - como cirugías a corazón abiertodel modernismo, este nuevo paradigma de revitalización busca curar el corazón urbano sin "crear grandes desarrollos urbanos, sino [arreglando] las pequeñas cosas que hacen que haya bienestar y calidad de vida" (Alcaldía de Bogotá, 2017). Estas pequeñas intervenciones - como agujas de acupuntura- se proponen como terapias para curar algunas de las "heridas" causadas por el modernismo. El PEMP, por ejemplo, propone el rediseño de la Carrera Décima, vía ampliada por los buldóceres del modernismo décadas atrás, como "un corredor verde del que nos sintamos orgullosos [pues se convirtió] en una herida que dividió [...] el centro" (El Tiempo, 2018). Así mismo, a diferencia del autoritarismo tecnocrático del modernismo, el PEMP está basado en extensos procesos de participación que incluyeron a residentes, comerciantes, estudiantes y hasta niños y niñas (IDPC, 2018). Sin embargo, la comunidad consultada no incluyó a vendedores informales ni habitantes de calle. Por el contrario, una de las conclusiones más repetidas en los diversos procesos de participación para definir la revitalización del centro resume la preocupación de diversos actores acerca de las "problemáticas sociales que [lo] afectan (inseguridad, habitantes de calle $[\mathrm{y}]$ ventas informales)" (IDPC, 2018, p. 33).

Esta preocupación específica sobre vendedores informales y habitantes de calle no es nueva. De hecho, los planes recientes de acupuntura urbana son la continuación de planes de revitalización de mucha mayor escala, que se basaron en el desplazamiento de estas poblaciones, vistas como agentes principales del deterioro del centro. El ejemplo más claro es el plan de renovación urbana de los barrios centrales de San Bernardo y Santa Inés. El proyecto fue justificado en 1998 como un esfuerzo de "recuperación y mejoramiento del sector, mediante [intervenciones] que le devuelvan la vitalidad urbana" (Alcaldía de Bogotá, 1998). Al igual que el PEMP, este programa estaba dirigido a recuperar zonas centrales percibidas como "desocupadas," "abandonadas" o carentes de "vitalidad." Sin embargo, en este y otros casos, la revitalización no se dirigía a curar la desocupación del sector. Por el contrario, la falta de vitalidad estuvo caracterizada por su ocupación por parte de una "concentración de indigentes y [...] otras territarias 42 
actividades como el comercio de drogas y otras [...] actividades ilícitas" (Alcaldía de Bogotá, 1998). Adicionalmente, haciendo referencia directa a la teoría de las ventanas rotas, la presencia de vendedores ambulantes y habitantes de calle fue considerada como una patología que, aunque no directamente criminal, debía ser extirpada de dichas zonas para prevenir otras patologías más graves (Donovan, 2008, 2010; Galvis, 2017b). Actualmente, bajo el PEMP, y a pesar del discurso acupuntural, se adelantó una operación similar en el sector aledaño del Voto Nacional. La administración buscaba dirigir a los habitantes de calle desplazados de allí a centros de atención fuera del espacio público, para lo cual urgió a "la comunidad [a no prestarles] ayudas [que] generan que [los habitantes de calle] no se quieran mover hacia nuestros planes de atención, pues les hace fácil la vida" (El Tiempo, 2016).

Así como el modernismo articuló ecos del urbanismo higienista, la revitalización urbana contemporánea también rescató algunos principios básicos del modernismo. En la Carta de Atenas, por ejemplo, Le Corbusier había propuesto que "la higiene basta para discernir los tugurios y discriminar los islotes claramente insalubres [que] deberán ser demolidos. Habrá que aprovechar esta circunstancia para sustituirlos por parques [que serán] el primer paso hacía la mejoración [sic] de las condiciones sanitarias" (Le Corbusier 1973, p. 69-70). El proyecto territarias 42 18
San Bernardo fue la demolición de varias manzanas alrededor de la llamada calle de El Cartucho, para dar paso, como lo proponía Le Corbusier, a la construcción del parque Tercer Milenio. Así mismo, en el barrio San Bernardo y sus alrededores y como parte del PZC, se planeó la construcción de un cluster de servicios hospitalarios llamado "Ciudad Salud," incluyendo hoteles de lujo y servicios comerciales para atraer turistas de la salud.

Si bien estas intervenciones urbanísticas a mayor escala hicieron escasas referencias explícitas a la acupuntura urbana, sus elementos básicos sí han formado parte de la filosofía de Bogotá y otras ciudades colombianas. De hecho, junto con los ejemplos de Curitiba y Barcelona - ambos claramente inspiradores de las intervenciones en Bogotá- los casos de Bogotá y Medellín son usados con frecuencia para ilustrar la acupuntura urbana. De hecho, como lo documenta Brand (2013) para el caso de Medellín, estas estrategias de acupuntura urbana han sido claves tanto para la adaptación local de dichos modelos como para la promoción internacional de la ciudad como ejemplo de innovación en la política urbana (Kapstein \& Ramírez, 2016). Más allá de la revitalización del centro, Bogotá ha empezado a proponer tácticas específicas de acupuntura para otras áreas de la ciudad.

El plan de desarrollo vigente desde 2016, por ejemplo, lista como parte importante de las "estrategías integrales de hábitat" el redesarrollo de sitios puntuales, argumentando que: 
[...] en algunos casos será mejor hacer micro intervenciones a nivel puntual $[\ldots]$ busca[ndo] el menor costo en estos procesos [de renovación urbana], Impuls[ando] procesos de 'acupuntura urbana' [que] embellezcan los espacios y promuevan su apropiación a través de la participación ciudadana" (Alcaldía de Bogotá 2016a, 1:197). En cuanto a la circulación, en vez de las grandes avenidas del modernismo, el plan propone la "conectividad de ciclo-rutas y alamedas [y la] acupuntura urbana para priorizar al peatón y al ciclista" (Alcaldía de Bogotá 2016a, p. 1:232).

En desarrollo de estas estrategias, la alcaldía propuso la creación de las "zonas de embellecimiento y apropiación (ZEA) [...] para recuperar la vitalidad de zonas [deterioradas]" (Alcaldía de Bogotá, 2016b). Intervenciones pequeñas y de bajo costo en estas zonas se proponen como una forma de "restaurar [sus] signos vitales [y] gener[ar] cambios positivos en los barrios; promov[iendo] acciones comunitarias que sean el punto de partida para el embellecimiento de la ciudad" (Alcaldía de Bogotá, 2016b).

Según esta propuesta de acupuntura urbana, la intervención restaurativa no tiene como único objeto el embellecimiento físico del área. Este, por el contrario, debe generar la activación de la ciudadanía y su apropiación del espacio público deteriorado. Al igual que las operaciones de revitalización de mayor escala del centro, el deterioro en los barrios no está ligado solamente a variables físicas. De hecho, la apropiación ciudadana de los espacios públicos con "signos vitales" restaurados tiene por objeto "garantizar que agentes generadores de inseguridad $\mathrm{y}$ desorden que han cooptado el espacio público sean desplazados" (Alcaldía de Bogotá, 2016b). Así, las ZEA buscan "mejorar las condiciones de seguridad [...] por medio del embellecimiento de los entornos y la apropiación de la comunidad [para] recuperar espacios obsoletos [y] desplazar paulatinamente las prácticas nocivas que afectan el entorno" (Concejo de Bogotá, 2017b).

Al igual que en la formulación del PEMP, la "cultura ciudadana" que la intervención acupuntual busca formar está limitada a una versión normativa de la comunidad, que incluye sobre todo a residentes y visitantes "legítimos," que son el sujeto preferido de apropiación. En este caso, la enfermedad urbana que se busca curar es la apropiación de espacios públicos por parte de "agentes de inseguridad y desorden," poblaciones patológicas que deben ser desplazadas no por el médico higienista ni por el buldócer modernista, sino por la comunidad empoderada por la activación acupuntural. Aprobadas por el Concejo Distrital en 2017, y ratificando los ecos de intervenciones urbanas de tiempos pasados, las ZEA funcionan como estrategias de embellecimiento y ornato junto a jornadas de limpieza similares a las establecidas un siglo atrás (Concejo de Bogotá, 2017a). territarias 42

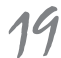




\section{Conclusiones}

A pesar de los esfuerzos para eliminar los "hábitos funestos" que devendrían en la degeneración racial de las clases populares, las autoridades higienistas nunca lograron erradicar la chicha en Bogotá ni consiguieron evitar la conformación de grandes barriadas populares. Igualmente, a pesar de las " 3 horas de bala y piedra" (El Vespertino, 1966a) y el "Plan contra Tugurios" que prometía "familia por familia erradica[r] el 'Policarpa" (El Vespertino, 1966b), los modernistas nunca consiguieron extirpar este "cáncer" específico. La facultad de medicina de la Universidad Nacional nunca consiguió construir su hospital. A pesar de los grandes recursos invertidos en renovar el barrio San Bernardo en el centro de Bogotá, "Ciudad Salud" continúa siendo un plan con poca perspectiva de ser realizado. Irónicamente, ninguna de estas intervenciones, usando metáforas médicas, logró sus objetivos, incluso cuando estos fueran efectivamente sanitarios. Sin embargo, todas estas intervenciones médicas en la ciudad consiguieron perpetuar el discurso según el cual ciertas poblaciones vulnerables constituyen un peligro para la ciudad en general y sus barrios y ciudadanos "sanos" en particular. En todos los casos, clases populares racializadas, inmigrantes rurales en barrios de invasión $\mathrm{y}$, últimamente, vendedores informales y habitantes de calle, han sido definidos como patologías. Así, su extirpación rutinariamente violenta, de los espacios urbanos que necesitan para vivir se ha justificado como una consecuencia necesaria de la protección general de la buena salud urbana.

A pesar de las continuas fallas de los diversos paradigmas planificadores, la persistencia de este discurso excluyente indica la continuidad histórica de un urbanismo centrado menos en responder a las necesidades básicas de las poblaciones más vulnerables que en defender los privilegios de las élites y clases medias urbanas contra las amenazas causadas por diversos elementos de contagio. De hecho, este urbanismo excluyente ha sido rutinariamente aplicado, como profilaxis médica, en nombre del bienestar general.

Si bien la muchos de los estudios críticos recientes sobre la revitalización urbana en Bogotá han mostrado las consecuencias excluyentes de estas políticas, también han tendido a mostrarlas como políticas que rompieron con la forma de pensar la ciudad en el pasado. Aunque los paradigmas de intervención urbana cambiaron, la exclusión subyacente a muchos de ellos puede verse como el resultado de continuidades históricas de larga duración.

Así, a pesar de articular discursos incluyentes y realizar prácticas de planeación participativa, estas intervenciones no deben ser juzgadas solo en términos de sus aspiraciones específicas. Después de todo, efectos esperados como los hospitales, las autopistas descongestionadoras o un concurrido parque Tercer Milenio, rara vez se 
han materializado en Bogotá. Por el contrario, estas intervenciones deben medirse en términos de los efectos diferenciados que producen sobre las poblaciones más vulnerables. Después de todo, muchas de estas políticas están nominalmente definidas como soluciones dirigidas precisamente a beneficiar a las mismas personas que terminan siendo desprovistas de los elementos más básicos de hábitat urbano.

\section{Referencias}

Acebedo, L. F. \& Moreno, O. (2003). Hernando Vargas vida y obra. Brunner era la academia, Le Corbusier la revolución urbanística. Bitácora Urbano Territorial, 1(7), 70-75.

Acero, V. H., Vargas, D., Bulla R. P., \& Cardona, O.S. (1997). Políticas saludables para la seguridad y la convivencia. Bogotá: Alcaldía Mayor.

Alcaldía de Bogotá. Decreto 185 de 1951. Por el cual se adopta el Plan Piloto de la ciudad y se dictan normas sobre urbanismo y servicios públicos $(5 \mathrm{de}$ abril de 1951).

Alcaldía de Bogotá. Decreto 880 de 1998. Por el cual se adopta el programa de Renovación Urbana para la recuperación del sector comprendido por los barrios San Bernardo y Santa Inés y su área de influencia y se establecen normas específicas para algunos de los sectores dentro del área de Renovación Urbana. (19 de octubre de 1998).
Alcaldía de Bogotá (2000). Acciones que recuperan la seguridad y la convivencia ciudadana. Bogotá: Secretaría de Gobierno.

Alcaldía de Bogotá (2016a). Plan Distrital de Desarrollo 2016-2020 "Bogotá Mejor para Todos”. Vol. 1. Bogotá: Alcaldía de Bogotá.

Alcaldía de Bogotá. Proyecto de Acuerdo 84 de 2016. Por el cual se crean las Zonas de Embellecimiento y Apropiación (ZEA) en el Distrito Capital y se dictan otras disposiciones. (Proyecto de acuerdo no promulgado).

Alcaldía de Bogotá (1 de marzo de 2017). Así es el plan de Peñalosa para renovar el centro de Bogotá. Boletín de prensa. Recuperado de http://www.bogota. gov.co/content/temas-de-ciudad/ cultura-y-recreacion/asi-es-el-plande-penalosa-para-renovar-el-centrode-bogota

Almandoz, A. (2007). Modernización urbanística en América Latina. Luminarias extranjeras y cambios disciplinares, 1900-1960. Iberoamericana, 7(27), $59-78$.

Ambler, W. H. (1985). Aristotle's Understanding of the Naturalness of the City. The Review of Politics 47(2), 163-185.

Amorocho, L., García, E., Angulo, J., \& Martínez, C. (1946). Bogotá puede ser una ciudad moderna. Revista Proa, (3), 15-25.

Anderson, W. (2006). Colonial pathologies: American tropical medicine, race, and territarias 42 


\section{territarias 42}

bygiene in the Philippines. Durham: Duke University Press.

Arango, L. D. (2018). Historia de una configuración profesional de urbanismo. Karl Brunner en Bogotá, 1933-1940. Cuadernos de Vivienda y Urbanismo, 11(22), 1-15.

Arango, L. D. (2019). Bogotá entre Marcel Poëte y Le Corbusier. La ciudad de Carlos Martínez. Bitácora Urbano Territorial, 29(1), 181-190.

Armus, D. (2007). La ciudad impura: salud, tuberculosis y cultura en Buenos Aires, 1870-1950. Buenos Aires: Edhasa

Atkinson, R. \& Gary, B. (Eds.) (2005). Gentrification in a global context: the new urban colonialism. New York: Routledge.

Beccassino, Á. (2000). Peñalosa y una ciudad 2600 metros más cerca de las estrellas. Bogotá: Grijalbo.

Beckett, K. \& Godoy, A. (2010). A Tale of Two Cities: A Comparative Analysis of Quality of Life Initiatives in New York and Bogotá. Urban Studies, 47(2), 277-301.

Berney, R. (2011). Pedagogical urbanism: Creating citizen space in Bogotá, Colombia. Planning Theory 10(1), 16-34.

Berney, R. (2017). Learning from Bogotá: Pedagogical Urbanism and the Reshaping of Public Space. Austin: University of Texas Press.

Bigon, L. (2016). Bubonic plague, colonial ideologies, and urban planning policies:
Dakar, Lagos, and Kumasi. Planning Perspectives, 31(2), 205-226.

Brand, P. (2013). Governing Inequality in the South through the Barcelona Model: 'Social Urbanism' in Medellin, Colombia. En Interrogating Urban Crisis: Governance, Contestation, Critique, De Montfort University. Recuperado de http://www.dmu. ac.uk/documents/business-and-lawdocuments/research/lgru/peterbrand.pdf

Brown, L. J., Dixon, D., \& Gillham, O. (2009). Urban Design for an Urban Century: Placemaking for People. Hoboken, NJ: Wiley.

Burdett, R., Cruz, T., Harvey, D., Sassen, S., \& Tehrani, N. (2014). Uneven Growth: Tactical Urbanisms for Expanding Megacities. New York: The Museum of Modern Art.

Calvo, I. Ó. I. \& Saade, G. M. (2002). La Ciudad en Cuarentena: Chicha, Patología Social y Profilaxis. Bogotá: Ministerio de Cultura.

Canguilhem, G. (2008). Knowledge of Life. Traducido por Stefanos Geroulanos y Daniela Ginsburg. New York: Fordham University Press.

Casagrande, M. (2015). Paracity: Urban Acupucnture. Volture, Italia: Oil Forest League.

Casanova, H. \& Hernández, J. (2011). The regeneration of public life. Scape, (1), 18-25. 
Casanova, H. \& Hernández, J. (Eds.) (2015). Public Space Acupuncture. New York: Actar.

Castro, M. J. (2013). El plano Bogotá Futuro. Primer intento de modernización urbana. Anuario Colombiano de Historia Social y de la Cultura, 40(2), 179-208.

Castro-Gómez, S. (2009). Tejidos oníricos: Movilidad, capitalismo y biopolitica en Bogotá, 1910-1930. Bogotá: Pontificia Universidad Javeriana.

Ceballos, O. L. (2008). Vivienda social en Colombia: una mirada desde su legislación, 1918-2005. Bogotá: Pontificia Universidad Javeriana.

Cervero, R. (2005). Progressive Transport and the Poor: Bogotá's Bold Steps Forward. ACCESS Magazine, 1(27), 24-30.

Chaparro, V. J., Pulido, M. B., \& Mendoza, D. M. (1997). Un siglo habitando los cerros: vidas y milagros de vecinos en el Cerro del Cable. Bogotá: Alcaldía Local Chapinero-Corporación RaícesIDCT. Recuperado de http://babel. banrepcultural.org/cdm/singleitem/ collection/p17054coll10/id/3744

Colombia, Congreso de la República. Ley 88 de 1947. Sobre fomento del desarrollo urbano del Municipio y se dictan otras disposiciones (26 diciembre 1947).

Colón, L. C. (2005). El saneamiento del Paseo Bolívar y la vivienda obrera en Bogotá. Revista Urbanismos, (2), 104-115.
Concejo de Bogotá. Acuerdo 3 de 1865. Orgánico de Cementerios ( 7 agosto 1865 ).

Concejo de Bogotá. Acuerdo 13 de 1880. Por el cual se establece un matadero público en la ciudad (22 mayo 1880). Concejo de Bogotá. Acuerdo 11 de 1881. Por el cual se crea una junta llamada del ramo de aguas.

Concejo de Bogotá. Acuerdo 14 de 1916. Por el cual se provee a la moralidad y salubridad de la capital de la República por medio de la reglamentación del expendio de bebidas embriagantes y se dictan otras medidas sobre el alcoholismo (16 junio 1916).

Concejo de Bogotá. Acuerdo 14 de 1917. Por el cual se provee a la pavimentación de las calles de la ciudad, especialmente la de las Avenidas Colón y de la República (31 marzo 1917).

Concejo de Bogotá. Acuerdo 15 de 1922. Sobre chichería (29 marzo 1922).

Concejo de Bogotá. Acuerdo 61 de 1922. Por el cual se introducen algunas modificaciones al artículo 2. del Acuerdo 15 de 1922 (chicherías) (16 diciembre 1922).

Concejo de Bogotá. Acuerdo 42 de 1928. Por el cual se aclaran los acuerdos números 15 de 1922, 18 de 1927 y 61 de 1922 y se dictan otras disposiciones (15 junio 1928).

Concejo de Bogotá. Acuerdo 697 de 2017. Por el cual se articulan acciones de embellecimiento y ornato en el Distrito Capital, a través de las Zonas de territarias 42

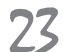




\section{territarias 42}

Embellecimiento y Apropiación (ZEA); se crea la campaña "Día Bogotá Limpia" y se dictan otras disposiciones (28 diciembre 2017).

Concejo de Bogotá. Proyecto de Acuerdo 103 de 2017. Por el cual se generan lineamientos para la conformación de Zonas de Embellecimiento y Apropiación (ZEA) en el Distrito Capital y se dictan otras disposiciones (04 diciembre 2015).

Crouch, D. (1998). Domesticating the street: the contested spaces of the high street and the mall. En N. Fyfe (Ed.), Images of the street: Planning, Identity, and control in public space (pp. 176191). London-New York: Routledge. Crump, J. (2002). Deconcentration by Demolition: Public Housing, Poverty, and Urban Policy. Environment and Planning D: Society and Space, 20(5), 581-596.

Cuéllar, M. \& Mejía, G. (2007). Atlas histórico de Bogotá. Cartografía 1791-2007. Bogotá: IDPC.

Donovan, M. (2008). Informal Cities and the Contestation of Public Space: The Case of Bogotá's Street Vendors, 19882003. Urban Studies, 45(1), 29-51.

Donovan, M. (2010). La Guerra por el espacio en Bogotá: La 'recuperacion' del espacio público y su impacto sobre los vendedores ambulantes. Territorios (12), 109-146.

Duque, F. I. (2011). Bogotá: entre la identidad y el marketing urbano. Cuadernos de Geografía-Revista Colombiana de Geografía, 20(1), 29-45.

El Espectador (26 de septiembre de 2016).

Distrito le apuesta a la recuperación y revitalización del centro histórico de Bogotá. El Espectador. Recuperado de https://www.elespectador.com/ noticias/bogota/distrito-le-apuestarecuperacion-y-revitalizacion-del-carticulo-656952

El Tiempo (13 de febrero de 1911). Higiene de Bogotá. El Tiempo.

El Tiempo (24 de febrero de 1929). Diversos Aspectos del Problema de la Higiene en Bogotá. El Tiempo.

El Tiempo (25 de junio de 1973a). Arquitectos Apoyan el 'Plan Cerros'. El Tiempo.

El Tiempo (25 de junio de 1973b). Campaña contra la 'chicha' en los barrios. El Tiempo.

El Tiempo (10 de agosto de 2016). Con \$35.000 millones será rehabilitado sector del 'Bronx' en Bogotá. El Tiempo. Recuperado de http://www. eltiempo.com/archivo/documento/ CMS-16670743

El Tiempo (27 de julio de 2018). El futuro de Bogotá está en un centro conectado y vibrante. El Tiempo. Recuperado de https://www.eltiempo.com/bogota/ el-plan-para-renovar-el-centro-debogota-en-los-proximos-anos-248812 El Vespertino (9 de abril de 1966a). La Batalla Campal en el Barrio 'Policarpa' 3 Horas de Bala y Piedra. El Vespertino. 
El Vespertino (13 de abril de 1966b). Plan Contra Tugurios. Familia por Familia será Erradicado el 'Policarpa'. El Vespertino.

Estrada, G. (2005). If You Build It, They Will Move: The Los Angeles Freeway System and the Displacement of Mexican East Los Angeles, 1944-1972. Southern California Quarterly, 87(3), 287-315.

Everett, M. (1998). Development Visions And 'Integration' On The Urban Frontier. Political and Legal Anthropology Review, 21(2), 1-10.

Foucault, M. (2008). Seguridad, territorio, población. Madrid: Ediciones AKAL.

Foucault, M. (2009). Nacimiento de la biopolitica: Curso del Collège de France (1978-1979). Madrid: Ediciones AKAL.

Galvis, J. P. (2014) Remaking Equality: Community Governance and the Politics of Exclusion in Bogotá's Public Spaces. International Journal of Urban and Regional Research, 38(4), 1458-1475.

Galvis, J. P. (2017a) Enrique Peñalosa. En R. Koch \& A. Latham (Eds.), Key Thinkers on Cities, $\mathrm{I}^{\text {ra }}$ edición (pp. 177-182). Thousand Oaks, CA: SAGE Publications Ltd.

Galvis, J. P. (2017b). Planning for Urban Life: Equality, Order, and Exclusion in Bogotá's Lively Public Spaces. Journal of Latin American Geography, 16(3), 83-105. Doi: https://doi. org/10.1353/lag.2017.0046
Gandy, M. (2014). The Fabric of Space: Water, Modernity, and the Urban Imagination. Cambridge: MIT Press.

Gehl, J. (2008). Life between buildings: using public space. Copenague: Danish Architectural Press.

Gehl, J. (2010). Cities for People. Washington: Island Press.

Goldberg, D. T. (1992). 'Polluting the Body Politic' Racist Discourse and Urban Location". En M. Cross \& M. Keith (Eds.), Racism, the City and the State (pp. 45-60). New York: Routledge.

Goossens, M. (2014). Jorge Gaitán Cortés y la introducción del urbanismo moderno en Colombia. Dearq, (14), 210-223.

Grupo de Estudios José Raimundo Russi (1975). Luchas de clases por el derecho a la ciudad: historia de las luchas de los barrios orientales de Bogotá, contra la Avenida de los Cerros. Bogotá: Editorial 8 de Junio.

Hawkins, M. (1997). Social Darwinism in European and American Thought, 1860-1945: Nature as Model and Nature as Threat. Boston: Cambridge University Press.

Hofer, A. (2003). Karl Brunner y el urbanismo europeo en América Latina. Bogotá: El Áncora EditoresCorporación la Candelaria.

Holston, J. (2008). Insurgent citizenship: disjunctions of democracy and modernity in Brazil. Princeton: Princeton University Press.

Ibarra, M. (2016). Hygiene and public health in Santiago de Chile's urban 


\section{tersitarias 42}

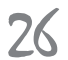

agenda, 1892-1927. Planning Perspectives, 31(2), 181-203.

Instituto de Capacitación para el Trabajo (ICT) (1964). Informe al señor Ministro de Fomento para su memoria al Congreso Nacional. Bogotá: ICT.

Instituto Distrital de Patrimonio Cultural (IDPC) (2018). PEMP Plan Espacial de Manejo y Protección. Centro Histórico de Bogotá. Proceso de Participación. Comunicación y Divulgación con Actores Públicos, Privados y Comunitarios 2018. Propuesta Integral. Bogotá: IDPC. Recuperado de http://idpc.gov.co/ wp-content/uploads/2018/09/ Proceso-participacio\%CC\%81n-2018. pdf

Jacobs, J. (1961). The death and life of great American cities. New York: Vintage.

Janoschka, M., Sequera, J., \& Salinas, L. (2014). Gentrification in Spain and Latin America-a Critical Dialogue. International Journal of Urban and Regional Research, 38(4), 1234-1265.

Johnson, S. (2007). The Ghost Map: The Story of London's Most Terrifying Epidemicand How It Changed Science, Cities, and the Modern World. New York: Riverhead Books.

Kapstein, P. \& Ramírez, M. (2016). Regeneración urbana integrada: proyectos de acupuntura en Medellín. Revistarquis, 05(9), 85-103.

Kelling, G. L. (1996). Fixing Broken Windows: Restoring Order and Reducing Crime in Our Communities. New York: Martin Kessler Books.
Kingman, G. E. (2006). La ciudad y los otros, Quito 1860-1940: higienismo, ornato y policía. Quito: Flacso- Ecuador.

Kraut, R. (2002). Aristotle: Political Philosophy. New York: Oxford University Press.

Kurokawa, K. (2000). The Philosophy of Symbiosis: From the Age of the Machine to the Age of Life. En A. White (Ed.), Kisho Kurokawa, architect and associates: selected and current works (pp. 6-12). Victoria, Australia: Images Publishing.

Le Corbusier. (1973) Principios de Urbanismo. La Carta de Atenas. Barcelona: Editorial Ariel.

Lerner, J. (2014). Urban Acupuncture. Washington: Island Press.

Llorente, M. V. (2005). Otra mirada a la experiencia de seguridad y convivencia en Bogotá. Quórum: revista de pensamiento Iberamericano, (12), 110-125.

López, B. L. (2003). Construir ciudadanía desde la cultura: aproximaciones comunicativas al Programa de Cultura Ciudadana, Bogotá, 1995-1997. Bogotá: Alcaldía Mayor.

Ludwig, J. \& Kling, R. J. (2007). Is crime contagious? The Journal of Law and Economics, 50(3), 491-518.

Lydon, M., Garcia, A., \& Duany, A. (2015). Tactical Urbanism: Short-Term Action for Long-Term Change. Washington: Island Press.

Martin, G. (Ed.) (2007). Bogotá: el renacer de una ciudad. Bogotá: Planeta, IDPC. 
Martin, G., Ceballos, M., \& Ariza, C. (2004) Bogotá: anatomia de una transformación: politicas de seguridad ciudadana 1995-2003. Bogotá: Pontificia Universidad Javeriana.

Mejía, G. R. (2000). Los años del cambio: historia urbana de Bogotá, 18201910. Bogotá: Pontificia Universidad Javeriana.

Mohl, R. A. (2004). Stop the road: Freeway revolts in American cities. Journal of Urban History, 30(5), 674-706.

Mondragón, L. H. (2008). Arquitectura en Colombia 1946-1951, lecturas críticas de la revista Proa. Revista Dearq, (2), 82-95.

Montero, S. (2017a). Persuasive Practitioners and the Art of Simplification: Mobilizing the 'Bogotá Model' through Storytelling. Novos Estudos, 36(1), 59-75.

Montero, S. (2017b). Worlding Bogotá's Ciclovía: From Urban Experiment to International 'Best Practice'. Latin American Perspectives, 44(2), 111-131.

Mumford, E. (2002). The CIAM Discourse on Urbanism, 1928-1960. Cambridge: MIT Press.

Mumford, E. (2007). El discurso del CIAM sobre el urbanismo, 1928-1960. Bitácora Urbano Territorial 1(11), 96-115.

Muñoz, L. (2018). 'Recovering' public space and race: Afro-Colombian street vendors in Bogotá, Colombia. Environment and Planning C: Politics and Space, 36(4), 573-588.
Noguera, C. E. (1998). La higiene como política, barrios obreros y dispositivo higiénico: Bogotá y Medellín a comienzos del siglo Xx. Anuario colombiano de historia social y de la cultura, (25), 188-215.

Noguera, C. E. (2003). Medicina y Politica: discurso médico y prácticas higiénicas durante la primera mitad del siglo XX en Colombia. Medellín: Universidad Eafit.

Nye, R. A. (1985). The Bio-Medical Origins of Urban Sociology. Journal of Contemporary History, 20(4), 659-675.

O'Byrne, O. M. C. (Ed.) (2010). LC BOG: Le Corbusier en Bogotá, 1947-1951. Bogotá: Universidad de los Andes.

Peck, J. (2005). Struggling with the Creative Class. International Journal of Urban \& Regional Research 29(4), 740-770.

Ritterbusch, A. E. (2016). Mobilities at Gunpoint: The Geographies of (Im) mobility of Transgender Sex Workers in Colombia. Annals of the American Association of Geographers, 106(2), 422-433.

Robledo, G. Á. M. \& Rodríguez, S. P. (2008). Emergencia del sujeto excluido: aproximación genealógica a la nociudad en Bogotá. Bogotá: Pontificia Universidad Javeriana.

Rodríguez, S. R., Jolly, J. F., \& Niño, S. A. (2004). Algunos apuntes sobre causas e indicadores del deterioro urbano: contribuciones a un debate sobre "vitalidad urbana”. Bogotá: Pontificia Universidad Javeriana. territarios 42

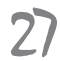


Sagaris, L. (2014). Citizens' Anti-highway Revolt in Post-Pinochet Chile: Catalyzing Innovation in Transport Planning. Planning Practice of Research, 29(3), 268-286.

Salazar, F. C., Mojica, R. X., \& Urrea, U. T. (2015). Urban Action. Under Cobblestones... The Beach! Dearq Revista de Arquitectura, 16(julio), 10-29.

Saldarriaga, R. A. (Ed). (1995). Medio siglo de vivienda social en Colombia 19391989. Bogotá: Inurbe.

Saldarriaga, R. A. (1996). Estado, ciudad y vivienda: urbanismo y arquitectura de la vivienda estatal en Colombia, 19181990. Bogotá: Inurbe.

Sánchez, A. J. M (2008). La biología humana como ideología: el racismo biológico y las estructuras simbólicas de dominación racial a fines del siglo XIX. THEORIA. Revista de Teoria, Historia y Fundamentos de la Ciencia, 23(1), 107-124.

Schnitter, P. (2007). Jose Luis Serty Colombia: De la Carta de Atenas a una carta del habitat. Medellín: Universidad Pontificia Bolivariana.

Sert, J. L. (1942). Can our cities survive?: An $A B C$ of urban problems, their analysis, their solutions. Cambridge: Harvard University Press.

Sevilla, B. Á., Castrillo, R. M. Á., Matesanz, P. Á., \& Sánchez, F. D. (2014). ¿Regeneración urbana? Deconstrucción

\section{territarias 42}

ecosociales y cambio global, (126), 129-139.

Shah, N. (2001). Contagious divides: Epidemics and race in San Francisco's Chinatown. Berkley: University of California Press.

Shatkin, G. (2011). Coping with actually existing urbanisms: The real politics of planning in the global era. Planning Theory 10(1), 79-87.

Simmel, G. (2004). The Metropolis and Mental Life. En M. Miles, T. Hall \& I. Borden (Ed.), The City Cultures Reader (pp. 13-19). New York: Routledge.

Solà-Morales, M. (2008). De cosas urbanas. Barcelona: Editorial Gustavo Gili.

Suárez, M. A. M. (2006). La ciudad de los elegidos. Crecimiento urbano, jerarquización social y poder politico. Bogotá (1910-1950). Bogotá: Editorial Guadalupe.

Swanson, K. (2007). Revanchist Urbanism Heads South: The Regulation of Indigenous Beggars and Street Vendors in Ecuador. Antipode, 39(4), 708-728.

Swanson, K. (2013). Zero Tolerance in Latin America: Punitive Paradox in Urban Policy Mobilities. Urban Geography, 34(7), 972-988.

Tarchópulos, D. (2006). Las huellas del plan para Bogotá de Le Corbusier, Sert y Wiener. Scripta Nova 10(218). Recuperado de http://www. ub.edu/geocrit/sn/sn-218-86.htm Vol. X, N. ${ }^{\circ} 218$ (86), 1 de agosto de 2006 
Thomas, D. (2016). Placemaking: An Urban Design Methodology. New York: Routledge.

Torres, C. A. (2013). La Ciudad en la Sombra: Barrios y luchas populares en Bogotá 1950-1977. Bogotá: Universidad Piloto.

Torres, G. M. (2001). Un psiquiatra decimonónico en el siglo xx. Miguel Jiménez López (1875-1955). Revista Colombiana de Psiquiatría, 30(2), 113-140.
Walljasper, J. (2007). The Great Neighborhood Book: A Do-it-rourself Guide to Placemaking. New York: New Society Publishers-Project for Public Spaces. Whyte, W. H. (2001). The social life of small urban spaces. New York: Project for Public Spaces.

Zambrano, F. (2009). Historia de Bogotá: Siglo Xx. Vol. III. Bogotá: Villegas Editores. 